\title{
Intrusion Detection System from External Threats using Data Mining
}

\author{
D. Parameswari, V.Khanaa
}

\begin{abstract}
Network Intrusion Detection is a significant apparatus to distinguish and examine security dangers to a correspondence arrange. It supplements other system security procedures, for example, firewalls, by giving data about the recurrence and nature of assaults. A system interruption discovery framework (NIDS) frequently comprises of a sensor that examines each bundle on the system under perception, and advances the parcels which are considered fascinating, together with an alarm message to a backend framework, that stores them for further examination and relationship with different occasions. The assessment procedure of the MAC address contrasted with the CADL is improved and streamlined with the help of the $\mathbf{J 4 8}$ choice tree calculation. The pursuit procedure is completed in the created arrangement esteem through tree based characterization.
\end{abstract}

Keywords:: MAC,CADL,CART

\section{INTRODUCTION}

The principle goal of J48 choice tree calculation is to limit the hunt procedure in contrast and the Current Active Directory List. The MAC address of the gadget is accessible in CADL. This rundown is utilized as a contribution to distinguish the gatecrasher. So as to recognize, it is proposed to utilize an adjusted J48 choice tree calculation. This calculation is likewise given a similar outcome tantamount to GA. Be that as it may, it tallies less time. The procedure stream of the changed J48 choice tree calculation is as per the following.

\section{Precondition} arranged in a climbing request and created list with one of a kind gadget portrayal of the system.

Step 1: The created MAC worth is assessed with the Current Active Directory List (CADL)

Step 2: The Current Active Directory list components are shaped as a tree dependent on the estimation of the current MAC address of the gadgets

Step3: At every hub left tree shaped with less worth components and right tree framed with more prominent worth components.

Step 4: Execute the stage 3 recursively till every one of the components in the CADL is incorporated into the tree.

Step 5: Evaluate the Left Significant Byte (LSB) to recognize the addition (Similarity) of the Device of the watched and suspected bundle created esteem.

Revised Manuscript Received on December 30, 2019.

* Correspondence Author

D. Parameswari, Research Scholar, Department of Computer Science and Engineering, Bharath Institute of Higher Education and Research

Chennai-600073, India. parameswariridu@gmail.com

Dr. V. Khanaa, Den-Info, Department of IT Bharath Institute of Higher Education and Research Chennai-600073,India, drvkannan62@yahoo.com

(c) The Authors. Published by Blue Eyes Intelligence Engineering and Sciences Publication (BEIESP). This is an open access article under the CC BY-NC-ND license (http://creativecommons.org/licenses/by-nc-nd/4.0/)
All the Current Active Directory List components are

Step 6: If the bits are equivalent at that point returns 1 generally 0 as an increase. aspect of the information mining to recognize the IDS.

Step 7: Count the increase esteem

Step8: If the addition most extreme worth is in the CADL then that gadget is validated device else prescribe for the speculated gadget. This procedure is executed as a major

\section{LITERATURE SURVEY}

Information mining is the way toward removing designs from information. Information mining is a significant apparatus by which present day business changes information into business insight giving an educational preferred position. It is as of now utilized in a wide scope of profiling rehearses. The most significant purpose behind utilizing information mining is to aid the investigation of accumulation and perception of conduct. Information digging innovation is progressed for preparing enormous measures of information, and to find covered up and disregarded data. Information mining generally includes four classes of bunching, arrangement, relapse and affiliation. Bunching is the undertaking of finding "comparable" gatherings and structures in the information, without utilizing the known structures. Characterization is the assignment of summing up realized structure to apply to new information. Normal calculations incorporate choice tree adapting, closest neighbor, Naive Bayesian grouping [1][2], neural systems and bolster vector machines. Relapse endeavors to discover a capacity which models the information with the least blunder. Affiliation guideline learning scans for connections between factors. The information mining approach is utilized to decide the obscure intrigued design from the space applications. The system security specialists endeavored to decide the IDS utilizing different information mining strategies.

A choice tree plays out the order of a given information test through different degrees of choices to enable us to arrive at an official choice. Such a succession of choices is spoken to in a tree structure. The tree structure is utilized in characterizing obscure information records. A choice tree with a scope of discrete (representative) class marks is known as an arrangement tree, though a choice tree with a scope of nonstop (numeric) values is known as a relapse tree. Truck (Classification and Regressing Tree) is an outstanding project, utilized in the structuring of choice trees. Choice trees utilize the IDE3, C4.5 and CART calculations [3][4].

The IDE3 (Iterative Dichotomiser 3) choice tree calculation is presented in 1986 by Quinlan Ross [5][6]. It depends on Hunt's calculation, and is sequentially executed. Like other choice tree calculations the tree is developed in two stages; tree development and tree pruning.

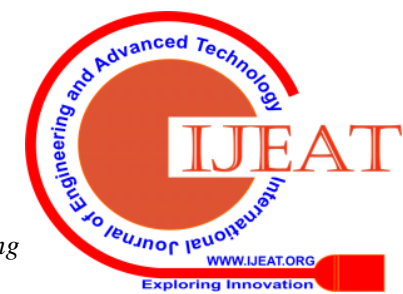




\section{Intrusion Detection System from External Threats using Data Mining}

Information is arranged at each hub during the tree building stage, so as to choose the best part single quality [7].

The IDE3 utilizes a data addition measure in picking the part property. It just acknowledges clear cut traits in structure a tree model [81][80].The IDE3 does not give exact outcomes when there is an excessive amount of clamor or subtleties in the preparation informational collection; subsequently a concentrated pre-handling of information is completed before structure a choice tree model with the IDE3.

The C4.5 calculation is an improvement of the IDE3 calculation, created by Quinlan Ross (1993). It depends on Hunt's calculation and like the IDE3, it is sequentially actualized. Pruning happens in C4.5 by supplanting the inside hub with a leaf hub, in this way decreasing the blunder rate (Podgorelecet al, 2002). In contrast to the IDE3, the C4.5 acknowledges both consistent and straight out characteristics in structure the choice tree. It has an improved strategy for tree pruning that decreases misclassification mistakes, because of commotion or an excess of detail in the preparation informational collection. Like the IDE3 the information is arranged at each hub of the tree, so as to decide the best part characteristic. It utilizes the increase proportion pollution technique to assess the part quality [8].

The CART (Classification and relapse trees) is presented by Breiman [9]. It manufactures both order and relapse trees. The characterization tree development via CART depends on the paired part of the characteristics. It is likewise founded on Hunt's model of choice tree development, and can be executed sequentially [9]. It utilizes the gini list part measure in choosing the part trait. Pruning is done in CART by utilizing a segment of the preparation informational collection. The CART utilizes both numeric and straight out qualities for structure the choice tree, and has in-assembled highlights that manage missing traits.

The CART is not quite the same as other Hunt's based calculations as it is additionally utilized for the relapse examination, with the assistance of the relapse trees. The relapse investigation highlight is utilized in estimating a reliant variable (result), given a lot of indicator factors over a particular timeframe. It utilizes many single variable part criteria like the gini list, symgini, and so forth., and one multi-variable (direct mixes) in deciding the best part point and the information is arranged at each hub to decide the best part point. The direct blend part criteria is utilized during the relapse examination.

The SVM first maps the info vector into a higher dimensional component space, and after that gets the ideal isolating hyper-plane in the higher dimensional element space. A SVM classifier is intended for parallel order. The speculation in this methodology generally relies upon the geometrical attributes of the given preparing information, and not on the particulars of the info space [10]. This methodology changes the preparation information into an element space of a gigantic measurement.

It forms the information from the system, and portrays measures that are huge for oddity discovery. Fluffy rationale [11] is a type of many-esteemed rationale. It manages thinking, which is surmised instead of fixed and definite. As opposed to customary rationale hypothesis, where parallel sets have two-esteemed rationale: (genuine or false), fluffy rationale factors may have a reality esteem that extents in degrees somewhere in the range of 0 and 1.Fuzzy calculations have been effectively connected to an assortment of mechanical applications, including autos, independent vehicles, compound procedures, and apply autonomy. A fluffy framework [12] includes a gathering of semantic explanations dependent on master information. This information is for the most part as on the off chance that rules. A case or an item can be recognized by applying a lot of fluffy rationale rules, in view of the qualities' semantic qualities.

\section{PROPOSED WORK}

The 16 bit portrayal of the gadget MAC address is displayed in the Current Active Directory List. The J48 choice tree calculation looks at the standardized data gain that outcomes from picking a trait for part the information. To settle on the choice, the trait with the most elevated standardized data addition is utilized. At that point the calculation repeats on the littler subsets. The part method stops if all occasions in a subset have a place with a similar class.

At that point a leaf hub is made in the choice tree advising to pick that class. For this situation, the J48 choice tree calculation makes a choice hub higher up in the tree utilizing the normal estimation of the class. On the off chance that the produced LSB esteem in CADL and approaching convention gadget MAC address are same then the gadget is verified generally the gadget suggested for the gatecrasher.

The structure of the altered J48 choice tree is appeared in Figure1. The primary degree of the tree is a solitary header hub. It is only a pointer hub to its youngsters. The second degree of the tree has 2 sub trees named from 1 to 2 .

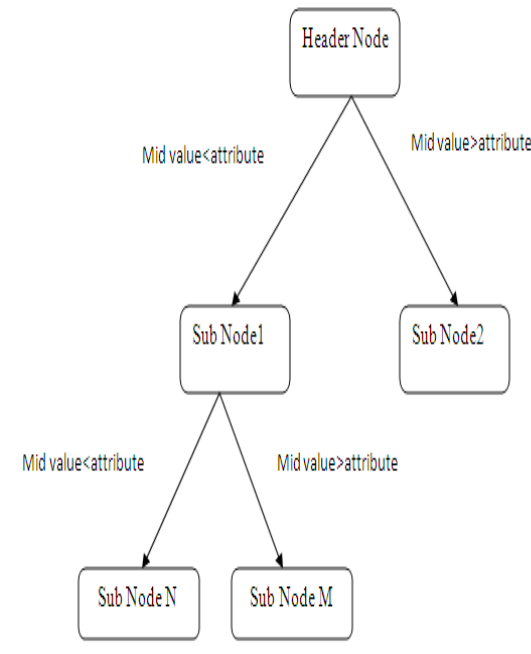

\section{Figure 1 Structure of the J48 decision tree}

Pseudo code for the J48 algorithm

The accompanying pseudo code is utilized to fabricate choice trees

1. Check for base cases [ Initial Device List structure Current Active Directory List]

2. For each property a\{ from the caught bundles Device address MAC\}

Discover the standardized data gain from part on a\{ select the 16 Bit gadget from the Least Most Significant Bit\} 
3. Let a best be the trait with the most noteworthy standardized data gain \{Allowed to convey on Network\}

4. Create a choice hub that parts on a best \{Select the Least Significant Bits or the significant piece for Cross Over\}

5. Recourse on the sub records gotten by part on a best, and include those hubs as offspring of hub

\section{EXPERIMENTAL RESULT}

. Give the tree a chance to speak to the maker OUI esteem and the gadget esteem, which is brought from the Current Active Directory List.

Level 0: Create a choice tree.

Level 1: The sub trees are created dependent on the qualities rather than the Index.

CADL List

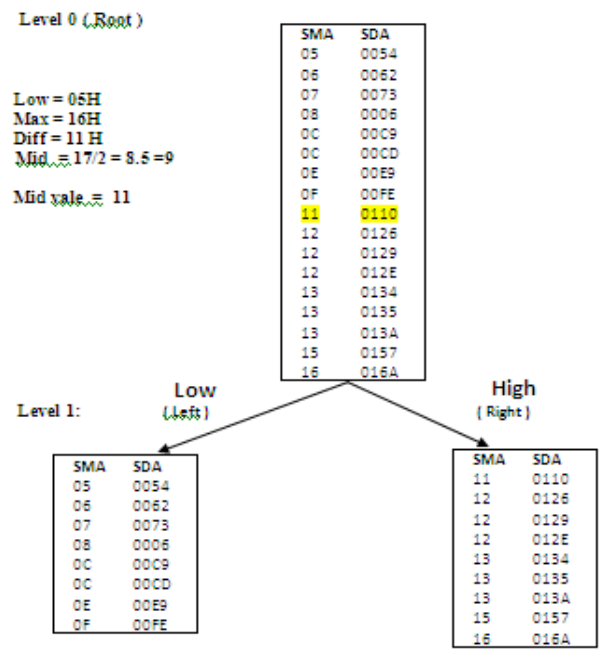

\section{Level 2:}

The parameter $08 \mathrm{H}$ is not exactly the mid worth 11.Therefore, the left tree is distinguished as a needy component and chose for further assessment.

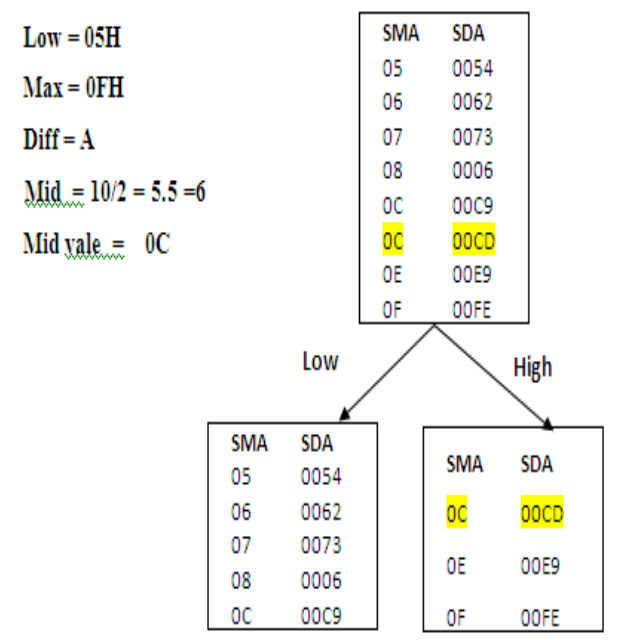

Level 3:

The parameter $08 \mathrm{H}$ is less than the mid worth $0 \mathrm{C}$. In this way, the left tree is distinguished as a needy component and chose for further assessment.

\begin{tabular}{|c|c|c|}
\hline Level 3 & SMA & SDA \\
\hline Low $=05$ & 05 & 0054 \\
\hline \multirow{2}{*}{$\mathrm{Max}=0 \mathrm{C}$} & 06 & 0062 \\
\hline & 07 & 0073 \\
\hline Diff $=07 \mathrm{H}$ & 08 & 0006 \\
\hline Vid vale $=7 / 2=3,5=4$ & $O C$ & $00 C 9$ \\
\hline
\end{tabular}

The mid worth is the fourth component $08 \mathrm{H}$. The component and the mid worth are the equivalent; along these lines, the component is recognized and suggested as a verified worth.

The presence of $08 \mathrm{H}$ is affirmed; along these lines, the arrival worth is 1 . It speaks to that the gadget imparted in the system is confirmed. The discovery result test is delivered beneath in Figure 5.2.

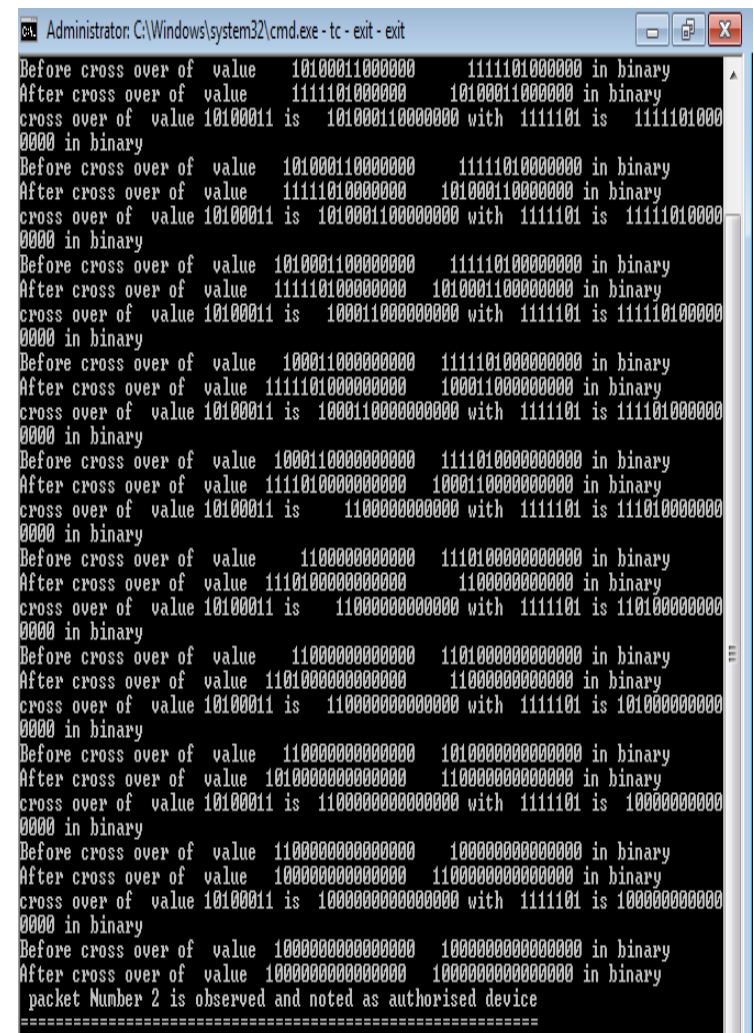

Figure 5.2 Intrusion Detection.

\section{CONCLUSION}

The quantity of records in the hubs is expanded; the quest time for the IDS is likewise expanded. The time multifaceted nature is unequal. In any case, with the J48 calculation, the time is similarly lesser than with the GA strategy, in light of the fact that the gadget reaches are resolved dependent on the worth file. On the off chance that the bundles are recognized as interruptions those gadgets are recorded as an associated gadget with the system; in this manner the normal time is less while assessing increasingly number of documents in high number of hubs in the system.

Published By:

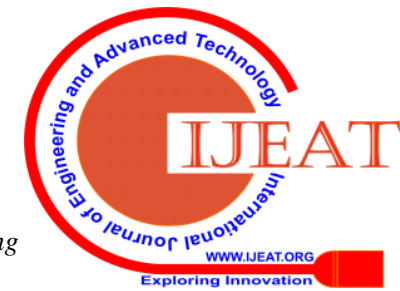




\section{Intrusion Detection System from External Threats using Data Mining}

\section{REFERENCES}

1. DewanMd, Farid, Mohammed Zahidur Rahman, “Anomaly Network Intrusion Detection Based on Improved Self Adaptive Bayesian Algorithm", Journal of Computers, Vol 5, pp-23-31, Jan 2010, DOI:10.4.304/jcp 5.1.

2. Panda M., and M. R. Patra, "Network intrusion detection using naïve Bayes," International Journal of Computer Science and Network Security (IJCSNS), Vol. 7, No. 12, December 2007, pp. 258-263.

3. Steinberg, D., and P. Colla. 1995. "CART: Tree-structured nonparametric data analysis”. San Diego, Calif., U.S.A.: Salford Systems.

4. Steinberg, D., P. Colla, and K. Martin. 1998. CART-Classification and regression trees: Supplementary manual for Windows. San Diego, Calif., U.S.A.: Salford Systems.

5. Quinlan, J.R. (1985b). "Decision trees and multi-valued attributes". In J.E. Hayes \& D. Michie (Eds.),Machine intelligence 11. Oxford University Press .

6. Quinlan, J.R. (1986). "Induction of decision trees. Machine learning" 1, 81-106.

7. Chen, Q., Aickelin, U. 2006. "Dempster-Shafer for Anomaly Detection". In Proceedings of the International Conference on Data Mining (DMIN 2006), Las Vegas, USA.

8. Quinlan J. R. "Discovering rules by induction In Expert Systems in the Micro-Electronic Age", Edinburgh University Press, 1993.

9. Breiman, L., Friedman, J., Olshen, R. and Stone, C. (1984) "Classification and Regression Trees", Wadsworth, Belmont, CA.

10. Goldberg, David E. "Genetic Algorithms in Search, Optimization, and Machine Learning”. New York, NY: Addison-Wesley, 1989.

11. Florez G., S.M. Bridges, and R.B. Vaughn, "An Improved Algorithm for Fuzzy Data Mining for Intrusion Detection”. The North American Fuzzy Information Processing Society Conference, New Orleans, LA, 2002.

12. Saniee M., Habibi J., Lucas C. "Intrusion detection using a fuzzy genetics-based learning algorithm". Journal of Network and Computer Applications, 30(1), pp. 414 - 428. January 2007.

13. Su-Yun Wu, and Ester Yen, "Data mining-based intrusion detectors," Expert Systems with Application`s, Vol. 36, Issue 3, Part 1, April 2009, pp. 5605-5612.

14. Taeshik Shon, Jong Sub Moon, "A Hybrid Machine Learning Approach to Network Anomaly Detection", Information Sciences 2007, Vol: 177, Issue: 18, Publisher: USENIX Association, pp- 37993821, ISSN:00200255,DOI:10.1016/j.ins-2007.03.025.

15. Tarek Abbes, Adel Bouhoula, MichaëlRusinowitch "Intrusion Detection Using Decision Tree", 2004 IEEE networks.

16. Teng.H.S, Chen.K and Lu.S.C, "Adaptive Real-Time Anomaly Detection using Inductively Generated Sequential Patterns", in the Proceedings of Symposium on research in Computer Security \& Privacy, IEEE Communication Magazine,1990, pp-278-284.

17. Vera Marinova-Boncheva, "A Short Survey of Intrusion Detection Systems", Institute of Information Technologies, 1113 Sofia, pp-2330, 2007.

18. Vern Paxson. Bro,A system for detecting network intruders in realtime, USENIX Security Symposium, San Antonio, TX, USA, January 1998.

19. W.Li. (2004) "Using Genetic Algorithm for network Intrusion Detection",Proceedings of the United States Department of Energy Cyber Security, USA.

20. Weiss W. R. and A. Baur. "Analysis of audit and protocol data using methods from artificial intelligence". In Proceedings of the 13th National Computer Security Conference, pages 109-114, Washington, D.C., USA, October 1990.

21. Whyte D., E. Kranakis, P. Van Oorschot"ARP - Based Detection of Scanning Worms within an Enterprise Network', In proce eding s of Annual Computer Security Applications Conference (ACSAC 2005) Tucson, AZ, Dec. 59, 2005.

22. Xiao. T, QU. G, Hariri. S, Yousif. M,“An efficient Network Detection Method Based on Information Theory and Genetic Algorithm", Proceedings of the 24th IEEE International Performance Computing and Communications Conference, Phonix, AZ, USA, 2005.

\section{AUTHORS PROFILE:}

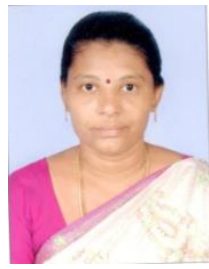

D. Parameswari, received his Master of Technology in Computer Science and Engineering from Bharath Institute of Higher Education and Research, Chennai. Currently she is working as Professor in Department of Information Technology, Jerusalem College of Engineering since 2002 and doing Research in the field of Network Security in
BIHER. Her areas of interests Networking, Data Mining, Cloud Computing.

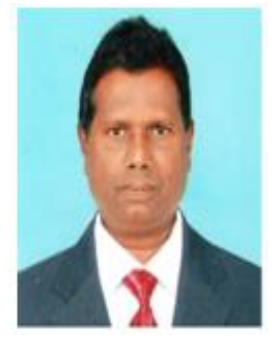

Dr. V. Khanaa, is self- directed, enthusiastic educator with a commitment on student development. He is with Bharath University, Chennai, Tamil Nadu, India as Professor and Dean of IT . He has over 35 years of rich experience in teaching along with student administration. He has guided more than 500 UG, PG projects and organized various national level conferences. He served as Senior Chair, Technical advisor in various national level conferences and Technical Committee member in International Conferences. He is an active member in CSI, IEEE, ISTE, ACM etc., His area of interests includes Computer Networks, Cloud Computing,Networks and Software Engineering. 